\title{
Underwater shock response analysis of a floating vessel
}

\author{
J.E. van Aanhold*, G.J. Meijer and \\ P.P.M. Lemmen \\ TNO Building and Construction Research, \\ Centre for Mechanical Engineering, P.O. Box 49, \\ 2600 AA Delft, The Netherlands
}

\begin{abstract}
The response of a surface vessel to underwater shock has been calculated using an explicit finite element analysis. The analysis model is two-dimensional and contains the floating steel structure, a large surrounding water volume and the free surface. The underwater shock is applied in the form of a plane shock wave and cavitation is considered in the analysis. Advanced computer graphics, in particular video animations, provide a powerful and indispensable means for the presentation and evaluation of the analysis results.
\end{abstract}

\section{Introduction}

The assessment of the vulnerability of naval vessels causes a continuous need for predicting the effect of underwater shock. Essential items to be considered in the analysis of ship structures subject to underwater shock are shock wave propagation effects during the early time response stage, fluid cavitation, inertia dominated fluid behaviour during the late time response stage and nonlinear structural response. In past analyses of structures subject to underwater shock, cavitation and nonlinear structural response were unfortunately too often neglected. Furthermore, the analysis of surface ships introduces an additional challenge.

This paper proposes a nonlinear finite element analysis based on a two-dimensional fluid/cavitation model for assessing the response of surface ship structures. Explicit time integration is used and the incoming shock wave is considered plane. This approach has an emphasis on early-time response, but the fluid

*Corresponding author. E-mail: J.vanAanhold@bouw.tno.nl. model is also capable of modelling the added hydrodynamic mass for the late-time response stage. The method provides the following advantages over other solutions:

- Suitable for the analysis of ship structures (primarily cross sectional deformations);

- Better description and understanding of cavitation;

- Capability to model bulk and hull cavitation in one and the same model;

- Extension toward nonlinear analysis;

- Improved assessment of the shock environment;

- Inherent transition to late-time response;

- Because of its 2D nature, acceptable in pre- and postprocessing effort and computing times;

- Possible extension toward $2 \frac{1}{2} \mathrm{D}$ analysis of a ship section (strip theory) and/or simplified 3D analysis of a ship section.

In order to demonstrate the feasibility of this method, first a number of simple one- and twodimensional test problems was analyzed. This was supported by theoretical studies regarding modelling techniques, convergence and stability. This paper describes the last subject of this study, the analysis of a floating vessel, with an emphasis on the behaviour of the cavitating fluid. All analyses were done using the public domain version of DYNA3D (Whirley and Hallquist [4]).

\section{Underwater explosion analysis method}

DYNA3D provides a fluid "material model" which may be used in combination with the standard 8-node solid brick element. This means that the hydrodynamic behaviour of the fluid following the acoustic wave equation is modelled using a standard Lagrangian displacement formulation. Although zeroenergy deformation modes, also known as "hourglassing" are suppressed, the absence of shear stiffness in a fluid still involves a serious risk that either excessive 
hourglassing occurs or that significant shear stresses develop as a result of hourglassing control. Both phenomena can lead to unacceptable results. Hence, the available fluid analysis options had to be well tested for this application. This displacement formulation has the advantage that the fluid can be coupled directly to the structure, but also increases the risk of excessive distortions of fluid elements near the fluidstructure interface. Because a fluid cannot transfer shear stresses, coupling of fluid and structure in tangential direction results in a small mass increase of the structure.

The volumetric constitutive behaviour of the water is described by the bilinear equation-of-state

$$
p=\max \left(-\rho_{\mathrm{w}} c_{\mathrm{w}}^{2} \varepsilon_{\mathrm{v}}, 0\right),
$$

where $p$ is the total pressure, $\varepsilon_{\mathrm{v}}$ the volumetric tensile strain, $c_{\mathrm{W}}$ the sonic velocity $(1450 \mathrm{~m} / \mathrm{s})$ and $\rho_{\mathrm{w}}$ the density $\left(1000 \mathrm{~kg} / \mathrm{m}^{3}\right)$. Cavitation is thus modelled by a zero fluid bulk stiffness for positive volumetric strains. The deviatoric constitutive behaviour of the water is described by means of a hydrodynamic viscosity coefficient.

With the exception of the free surface, the outer boundary of the water mesh is modelled as a nonreflecting boundary. The plane step-exponential shock wave is modelled by means of pressure time histories which act on this boundary. These pressure time histories are corrected for surface cutoff effects. The constitutive behaviour of the fluid also covers the added hydrodynamic mass effects which are important in the late time response stage. A necessary condition is that the nonreflecting model boundaries are at sufficient distance from the structure, so that added mass effects and cavitation are described properly by the fluid contained within the model. This approach avoids the need for implementing approximations such as the Double Asymptotic Approximation (DAA) at the nonreflecting boundary (Felippa and de Runtz [2]). Because of its Lagrangian nature, this method cannot properly describe fluid flow phenomena.

\section{Test problems}

The first test problem that was analyzed is the onedimensional problem of a plate floating on the water surface loaded by an underwater shock as described by Bleich and Sandler [1]. This example, which is often referred to in literature, was used as a first test. Various analyses using one- and two-dimensional models were done and their results are in good accordance with those by Bleich and Sandler [1]. No hourglassing problems were encountered. The main problem appeared to be dispersion, i.e., a shock wave looses its steepness during its propagation.

The second test problem was the two-dimensional problem described by Felippa and DeRuntz [2], a modified version of the problem originally analyzed by Newton [3]. This case consists of a deepwater cylindrical shell of $10 \mathrm{~m}$ diameter in an infinite fluid domain loaded by a plane step-exponential shock wave. Also here the results agree well with those by Felippa and DeRuntz [2]. Again the solution suffered somewhat from dispersion, but it must be emphasized that also the original results by Felippa and DeRuntz [2] exhibit significant dispersion effects.

In order to verify whether the fluid model correctly accounts for added hydrodynamic mass effects, an extra analysis was done where the cylindrical shell of the previous test problem was fixed with relatively soft node-to-ground springs. This analysis was run for a long analysis time, i.e., a few seconds. The effective added mass as estimated from the resulting natural frequency associated with the rigid body motion showed that the added mass is described with sufficient accuracy.

The conclusions of these tests were sufficiently encouraging to continue this study with a realistic structure.

\section{Analysis model of a floating structure}

The structure considered in this study consists of a cylindrical shell which is floating on the water surface. The shell has a radius $1.55 \mathrm{~m}$ and a thickness $25 \mathrm{~mm}$. A spring-supported rigid deck is mounted inside the shell. The draught is $1.55 \mathrm{~m}$. The structure is loaded by a heavy step-exponential shock wave which travels at an angle of 45 degrees with the horizontal plane. The decay time $\theta$ is approximately $1 \mathrm{~ms}$. Only linear elastic structural behaviour was considered in the current analysis. A number of analyses has been performed using different finite element models, including some for a fluid domain without the structure.

The analysis presented here has a surrounding water domain of $15 \mathrm{~m}$ width and $10 \mathrm{~m}$ depth. These dimensions were based on the expected cavitation region and the need for a proper description of added hydrodynamic mass effects. The average element size 


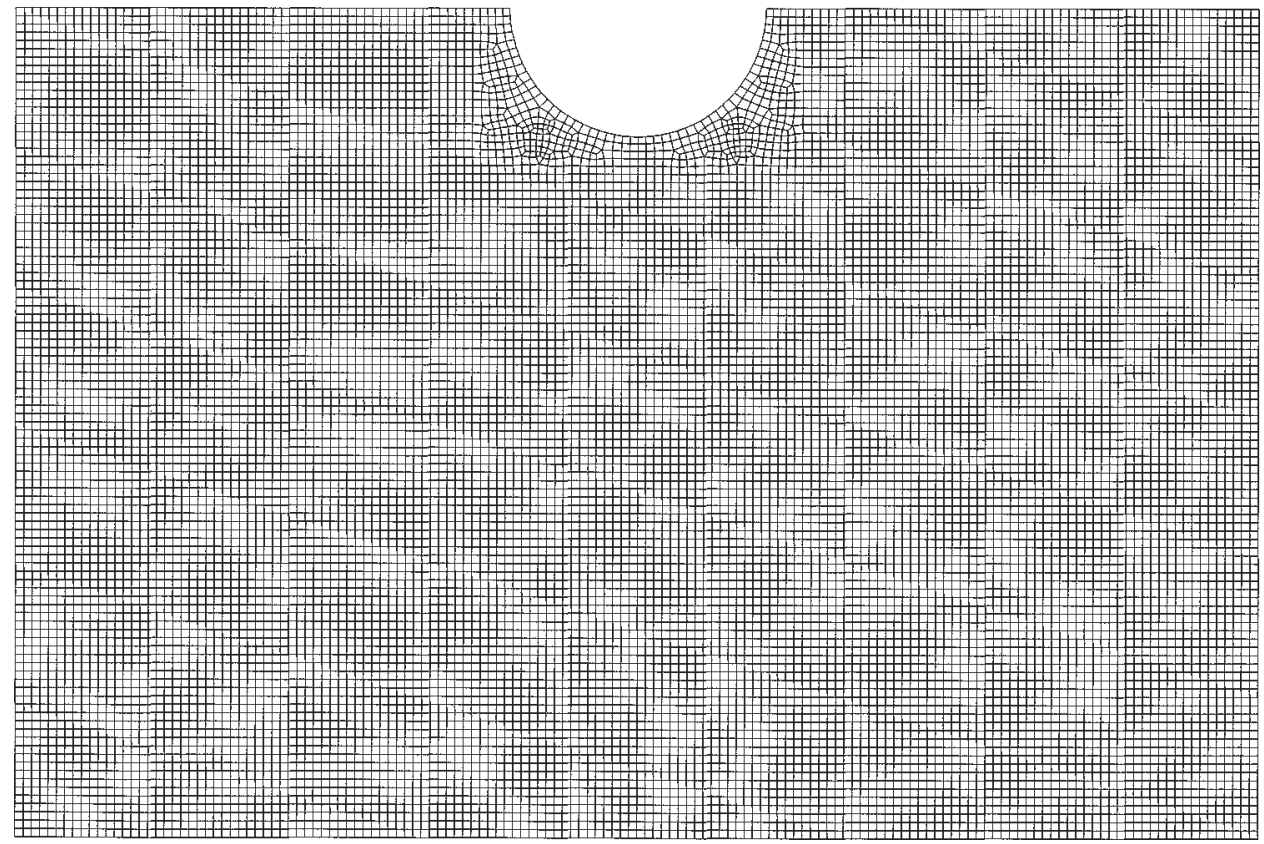

Fig. 1. Finite element model of fluid domain.

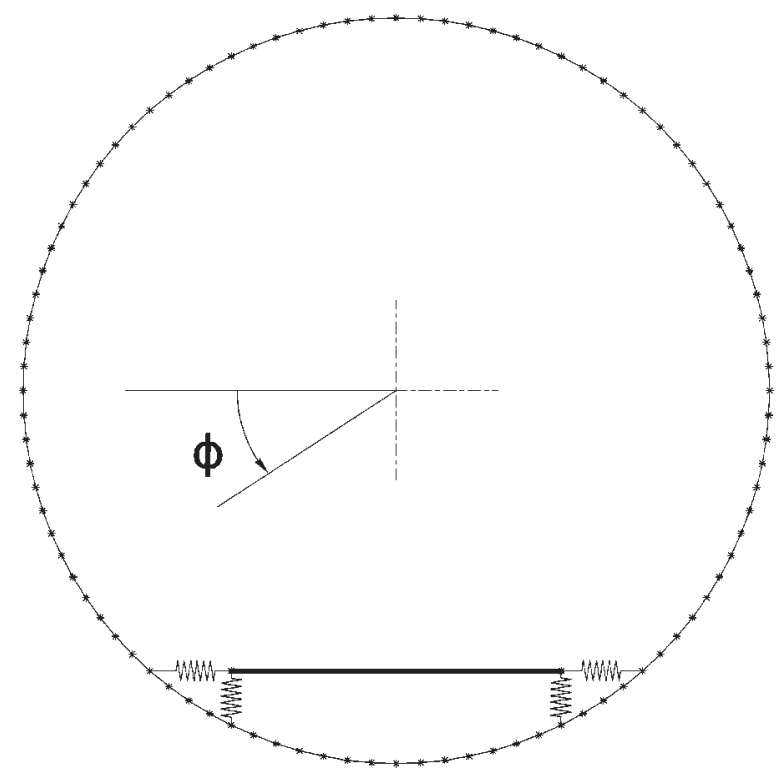

Fig. 2. Structural finite element model.

$\ell_{\text {element }}$ is $0.1 \mathrm{~m}$, which corresponds to a dimensionless element length

$$
\alpha=\frac{\ell_{\text {element }}}{c_{\mathrm{w}} \theta} \sim 0.07
$$

where $\theta$ is the decay time of the step-exponential shock wave. Theoretical considerations showed that this dimensionless element size provides a reasonable optimum between model size and minimization of dispersion and spurious oscillations within the solution. A lesson learned from other analyses is that a regular mesh yields favourable results. Hence, the mesh contains mainly cube-shaped elements except for some inevitable slightly distorted elements near the structure. The finite element model of the water is shown in Fig. 1, the model of the shell and spring-supported deck in Fig. 2.

The total mesh consists of 30104 nodes, 14694 solid fluid elements, 96 shell elements for the shell structure, 1 shell element for the rigid deck and $2 \times 4$ springs. A total of 350 nonreflecting boundary elements have been applied for modelling the bottom, left and right side of the fluid mesh. The mesh has a thickness $0.1 \mathrm{~m}$ in transverse direction. The shock wave enters the model at zero time at the left bottom corner nodes of the mesh and propagates at a $45^{\circ}$ angle in right-upward direction.

\section{Analysis}

The analysis was done for two different durations:

(a) An early time response analysis until $15 \mathrm{~ms}$ with an output resolution of $0.05 \mathrm{~ms}$. Output are the initial coordinates, initial pressures, displacements and volumetric strains. These data 
are output as binary datafiles and add up to $314 \mathrm{Mb}$ of data. The analysis used 1552 time steps with an almost constant time step of approximately $10 \mu$ s and almost 1 hour CPU time on a Silicon Graphics work station.

(b) A cavitation closure analysis until $400 \mathrm{~ms}$ with an output resolution of $1 \mathrm{~ms}$. Similar binary output datasets add up to no less than $406 \mathrm{Mb}$ of data. This analysis used 41378 time steps with an almost constant time step of approximately $10 \mu$ s and approximately 21 hours CPU time.

\section{Presentation of results}

The vast amount of output generated by DYNA3D created the need to spend much effort on the presentation of results. Earlier analyses showed that the calculated fluid behaviour may be somewhat disturbed by the occurrence of spurious cavitation. Presentation of results in the form of usual pressure contour plots does not allow to separate real and spurious cavitation. It offers advantages to consider the volumetric tensile strain $\varepsilon_{\mathrm{v}}$ instead. It was discovered that spurious cavitation usually goes together with insignificant volumetric tensile strains against much larger values for real cavitation. However, the volumetric compressive strains at the shock wave are very low compared to the maximum volumetric tensile strain, which is 0.19 for the analysis presented here. The optimum solution is to use pressure/cavitation contour plots where pressures in non-cavitating regions are plotted by real colours (57 colours ranging from dark blue for zero pressure through red for peak explosion pressure), whilst in cavitating regions volumetric tensile strains are plotted using gray scales (7 gray scales ranging from dark for the cavitation threshold through light for maximum volumetric tensile strain using different contour increments). The threshold between spurious and real cavitation is set to $1 / 1000$ times the maximum volumetric tensile strain, which makes spurious cavitation invisible. Such pressure/cavitation plots using the deformed mesh are generated using MATLAB on a Silicon Graphics work station for every output time step and saved as TIFF graphics format files. This required approximately 30 hours CPU time and $127 \mathrm{Mb}$ more disk storage. Finally, Silicon Graphics animation and video manipulation software was used to generate a 400 frames video animation from these pictures.

\section{Analysis results for water}

The results were evaluated using a video animation which consists of a series of pressure/cavition contour plots on the deformed water mesh, where the deformations have been exaggerated by a factor 5 . Figures 3 through 6 show four examples of such plots.

Figure 3 shows the deformations and pressure/cavitation contours at $8 \mathrm{~ms}$. The shock wave, which shows already some dispersion, has reached the surface and the structure, resulting in a large area of bulk cavitation. Note the thin layer of non-cavitating elements on top of the bulk cavitation. The water surface is raising from the left.

Figures 4 and 5 show the deformations and pressure/cavitation contours at 22 and $60 \mathrm{~ms}$, respectively, with large shell deformations, large deck deflections and an increasing bulk cavitation area. These figures reveal minor spurious shear deformations in the fluid along vertical lines aside the structure.

The bulk cavitation area remains increasing and the water surface raising until approximately $96 \mathrm{~ms}$. At the same time, the thickness of the water layer on top of the bulk cavitation is increasing. The maximum vertical displacement of the water surface is $0.34 \mathrm{~m}$ against an average maximum vertical displacement $0.25 \mathrm{~m}$. There is a continuous opening and closure of small cavitation regions near the hull. The water surface shows some small waves. Beyond $96 \mathrm{~ms}$, the thickness of the bulk cavitation layer decreases while the thickness of the water layer on top of it increases. The bottom of the cavitation remains approximately at the same location. After approximately $150 \mathrm{~ms}$ the bulk cavitation layer starts to show necking somewhat to the right underneath the hull. The bulk cavitation starts to close at $190 \mathrm{~ms}$ at the same location, which is shown in Fig. 6.

From this moment onward, there are two bulk cavitation regions which close like a zipper toward the nonreflecting boundaries of the model. This cavitation closure goes together with the generation of shock waves which are cylindrical because of the 2D nature of the analysis. The resulting reloading pressures at the hull between 180 and $220 \mathrm{~ms}$ are low compared to the primary shock waves. After this moment some less significant hull cavitation continues until the end of the analysis (400 ms).

It may be discussed whether the observed cavitation closure behaviour is realistic. In an additional analy- 


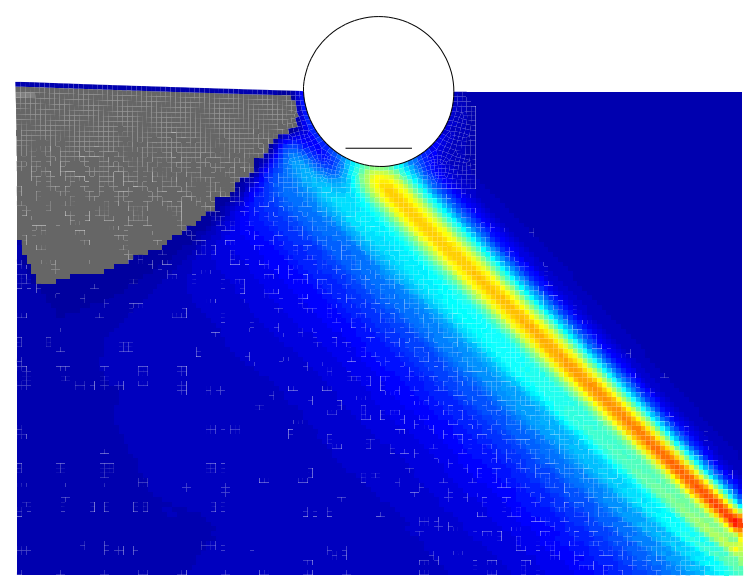

Fig. 3. Pressure/cavitation contours and deformations for $8 \mathrm{~ms}$.

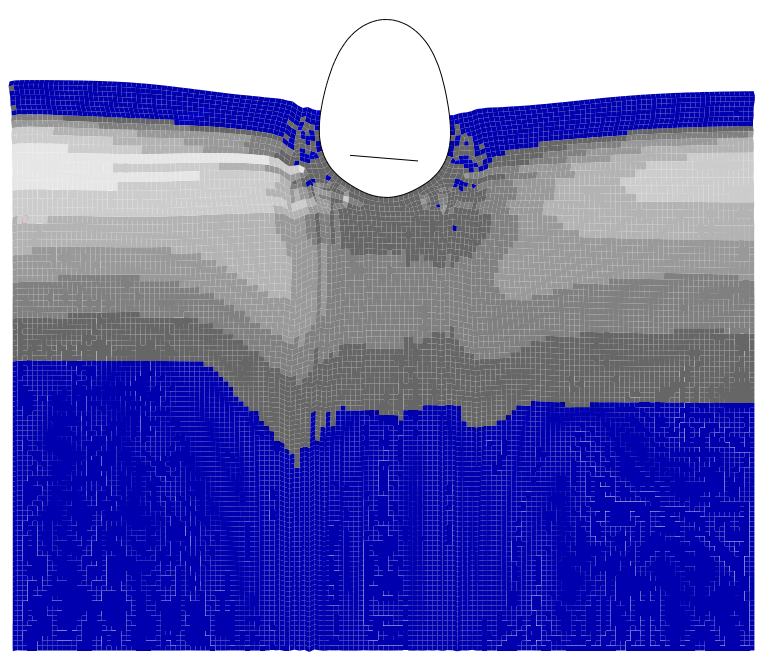

Fig. 5. Pressure/cavitation contours and deformations for $60 \mathrm{~ms}$.

sis with a fully regular mesh of water only (without a structure), the caviation closed as expected like a zipper travelling from the left to the right at $210 \mathrm{~ms}$, i.e., in the same direction as the shock wave passed by. On the other hand, a strong mesh dependency of the position and shape of the cavitation regions was found for more irregular meshes. It must nevertheless be concluded that presence of the structure shortens the duration of bulk cavitation.

Figure 7 shows pressure histories along the hull. Shock wave arrival is visible at approximately $8 \mathrm{~ms}$. Bulk cavitation is shown as flat areas. The figure also shows the reloading pressures resulting from bulk cavitation closure. Hull cavitation closure yields lo-

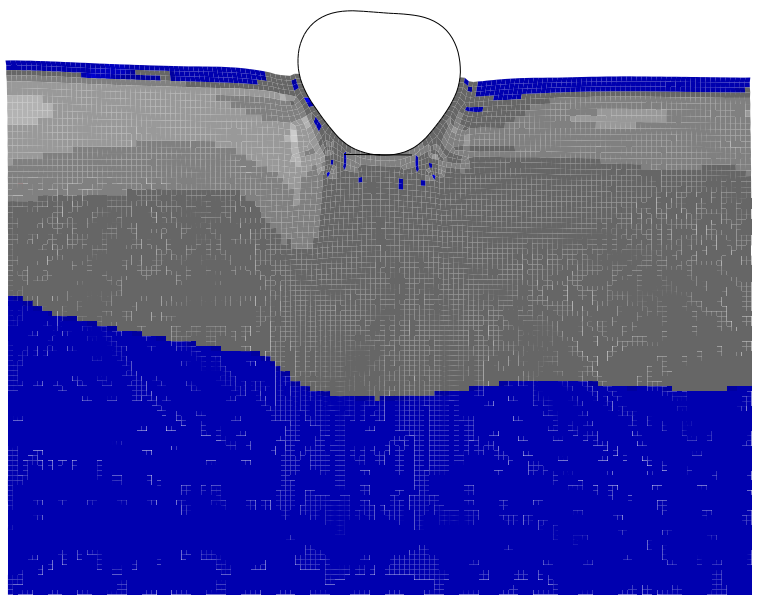

Fig. 4. Pressure/cavitation contours and deformations for $22 \mathrm{~ms}$.

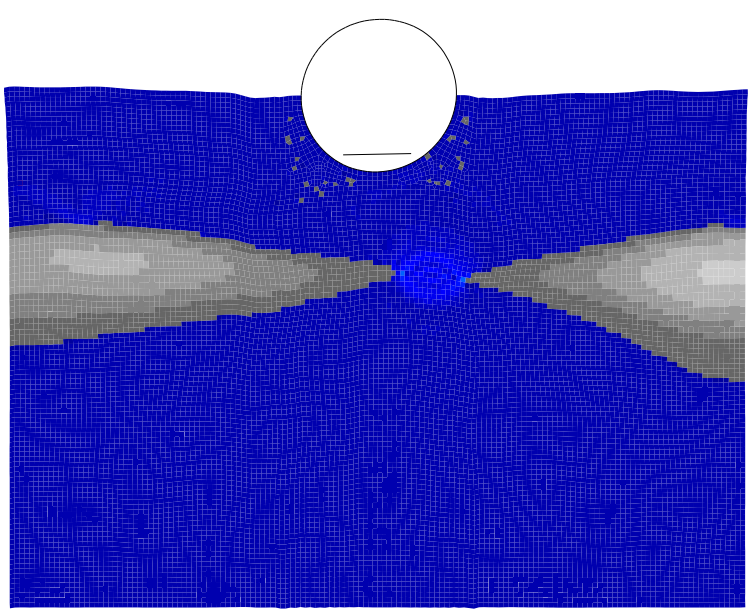

Fig. 6. Pressure/cavitation contours and deformations for $190 \mathrm{~ms}$, showing cavitation closure.

cal pressure peaks which are also of minor importance.

Figure 8 shows the maximum hull pressures for shock wave arrival (7-10 ms) and reloading (180$220 \mathrm{~ms})$. This shows that the maximum pressures are found at the location of first shock wave arrival $\left(\phi=45^{\circ}\right)$ and just before the location where the shock wave strikes the hull tangentially $\left(\phi=135^{\circ}\right)$. The maximum hull pressure is approximately $60 \%$ of the peak explosion pressure. The maximum pressures in the shadow zone beyond $\phi=135^{\circ}$ are considerably lower. Furthermore, the maximum hull pressures during reloading are low compared to those during shock wave arrival. 


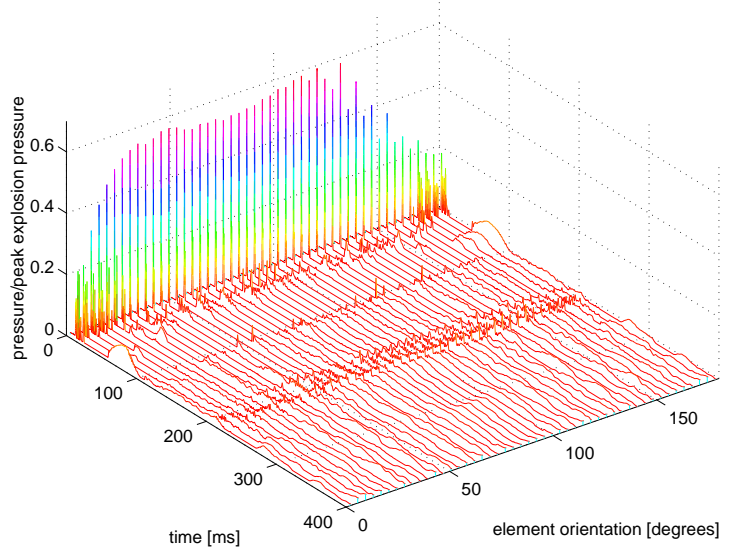

Fig. 7. Hull pressures as a function of time and position angle $\phi$.

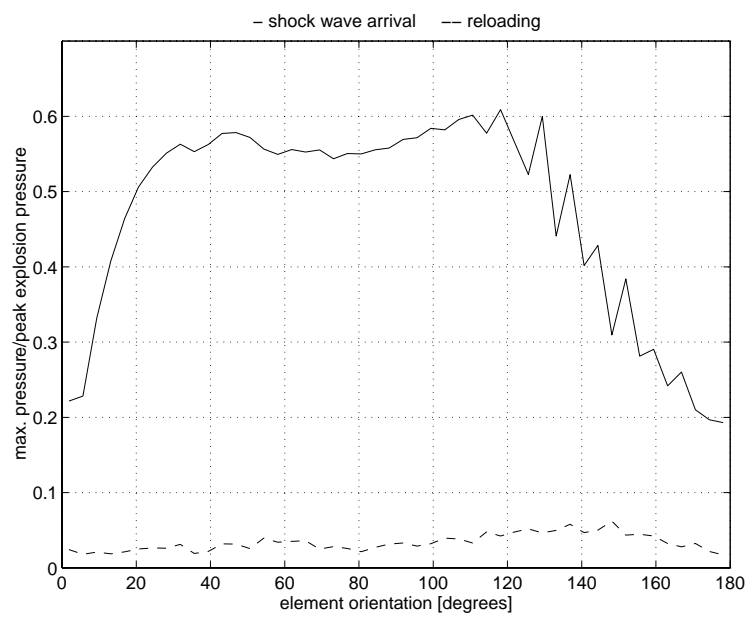

Fig. 8. Maximum hull pressures during shock wave arrival (solid line) and reloading (dashed).

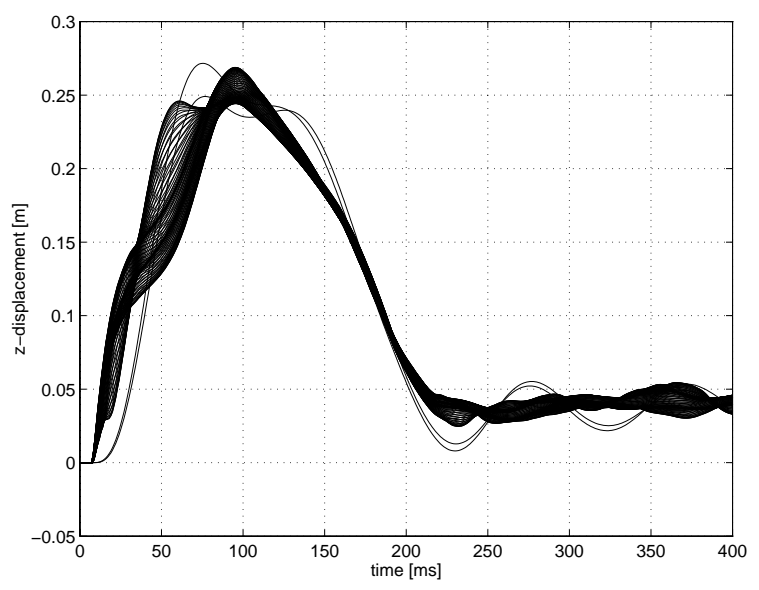

Fig. 9. Vertical displacements of hull nodes as a function of time.

\section{Analysis results for hull}

The hull moves predominantly in vertical direction. Figure 9 shows the vertical displacements of the hull nodes. The maximum vertical displacement is approximately $0.25 \mathrm{~m}$, which is mainly a rigid body translation. This value equals the average maximum displacement of the water surface. The horizontal rigid body motion is very small. The maximum deflections due to vibrations are $55 \mathrm{~mm}$. The maximum vertical spring deflection is $85 \mathrm{~mm}$.

\section{Conclusions}

It is concluded that the analysis method as presented in this paper is well suitable for this class of problems and produces results which are in reasonable agreement with solutions from literature. The presented demonstration analysis shows that it is feasible to calculate the underwater shock response of a surface vessel for the primary shock wave with bulk and hull cavitation. Advanced computer graphics, in particular video animations, provide a powerful and indispensable means for the presentation and evaluation of the results. Although some questions about the results remain, in particular about bulk cavitation closure, the results are very instructive and encouraging for future work. Most serious remaining problems are dispersion (a shock wave looses its steepness during its propagation) and the present-day practical hardware and software limits, which unfortunately still obstruct a full three-dimensional analysis of a complete naval ship structure. Further work needs to be done in addressing cavitation closure, the effects of a pulsating gas bubble and nonlinear elastic-plastic structural response. Finally, validation by means of full scale experiments remains indispensable.

\section{Acknowledgement}

The authors express their gratitude to the Royal Netherlands Navy for sponsoring the work presented in this paper.

\section{References}

[1] H.H. Bleich and I.S. Sandler, Interaction between structures and bilinear fluids, Int. J. Solids and Structures 6 (1970), 617-639. 
[2] C.A. Felippa and J.A. DeRuntz, Finite element analysis of shock-induced hull cavitation, Computer Methods in Applied Mechanics and Engineering 44 (1984), 297-337.

[3] R.E. Newton, Effects of cavitation on underwater shock loading - Plane problem, final report, Report no. NPS-69-81001A Naval Postgraduate School, Monterey, CA.
[4] R.G. Whirley and J.O. Hallquist, DYNA3D - A nonlinear, explicit, three-dimensional finite element code for solid and structural mechanics - User Manual, Report no. UCRLMA-107254 Lawrence Livermore National Laboratory, Livermore, CA.

Received 3 October 1997; Revised 4 February 1998 

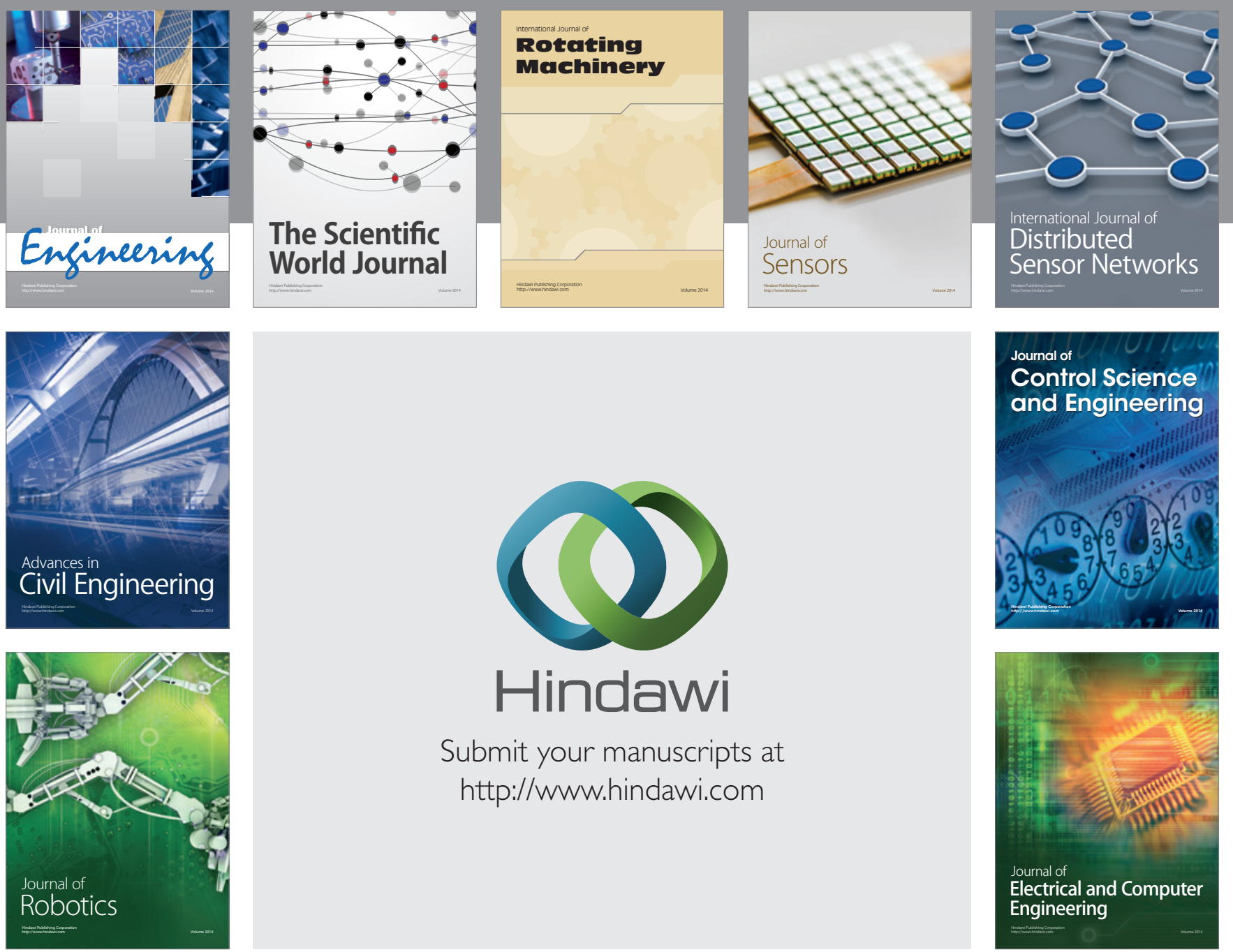

Submit your manuscripts at

http://www.hindawi.com
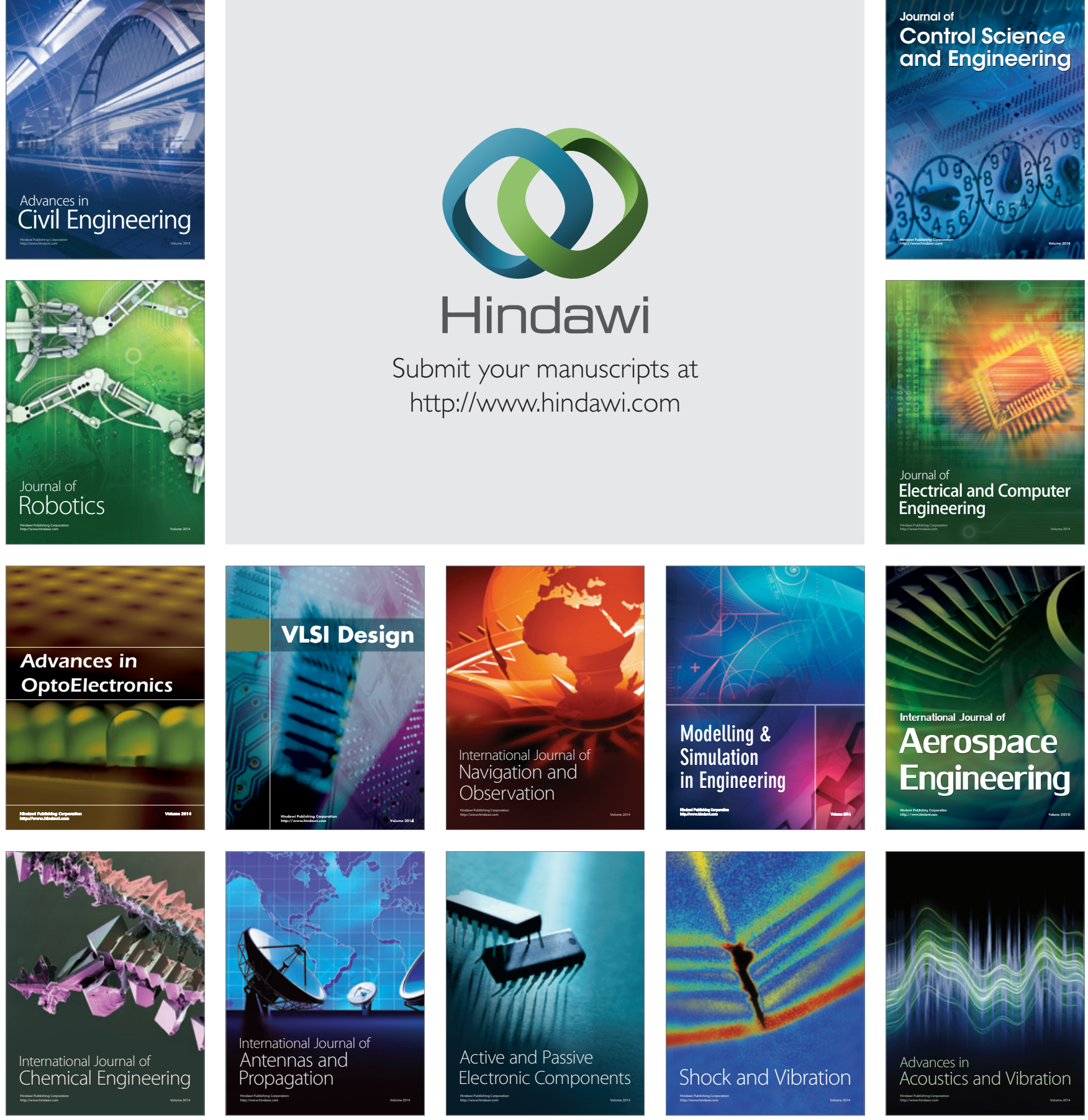